Brain and Language; Volume 167, April 2017, Pages 94-105

https://doi.org/10.1016/j.bandl.2016.02.005

\title{
The beneficial role of memory reactivation for language learning during sleep: a review.
}

\author{
Thomas Schreiner ${ }^{1,2} \&$ Björn Rasch ${ }^{1,2}$ \\ 1 University of Fribourg, Department of Psychology, Fribourg, Switzerland \\ 2 Zurich Center for Interdisciplinary Sleep Research (ZiS), Zurich, Switzerland
}

Corresponding authors:

Thomas Schreiner, University of Fribourg, Department of Psychology, Division of Cognitive

Biopsychology and Methods, Rue P.-A.-Faucigny 2, CH-1701 Fribourg, Switzerland;

Email: thomas.schreiner@uzh.ch

Björn Rasch, University of Fribourg, Department of Psychology, Division of Cognitive

Biopsychology and Methods, Rue P.-A.-Faucigny 2, CH-1701 Fribourg, Switzerland;

Email: Bjoern.Rasch@unifr.ch

Phone: +4126300 7637

Keywords: sleep, language learning, memory, reactivation 
Abstract

Sleep is essential for diverse aspects of language learning. According to a prominent concept these beneficial effects of sleep rely on spontaneous reactivation processes. A series of recent studies demonstrated that inducing such reactivation processes by re-exposure to memory cues during sleep enhances foreign vocabulary learning. Building upon these findings, the present article reviews recent models and empirical findings concerning the beneficial effects of sleep on language learning. Consequently, the memory function of sleep, its neural underpinnings and the role of the sleeping brain in language learning will be summarized. Finally, we will propose a working model concerning the oscillatory requirements for successful reactivation processes and future research questions to advance our understanding of the role of sleep on language learning and memory processes in general. 


\section{Introduction}

In today's multilingual world, interaction amongst people with diverse cultural background and mother tongues is a permanent feature. Thus, learning and mastering new languages becomes increasingly important, and potential approaches to facilitate and accelerate tedious language learning processes are highly desirable for many of us. According to recent findings, sleep might be an ideal state for the application of such learning enhancing approaches (Diekelmann, 2014). Sleep after learning generally promotes divers aspects of language learning, ranging from word learning to the abstraction of grammatical rules (Batterink, Oudiette, Reber, \& Paller, 2014; Henderson, Weighall, Brown, \& Gareth Gaskell, 2012). Furthermore, emerging techniques as targeted reactivation of language-related memory representations might fundamentally change how we learn foreign languages in the future.

In this review, we aim at presenting recent models and empirical findings concerning the beneficial effects of sleep on language learning, focussing particularly on studies that actively enhance specific aspects of language learning during sleep. Accordingly, the memory function of sleep and its role in language learning will be reviewed. Furthermore, we will summarize the ability of the sleeping brain to process auditory sensory input, constituting a fundamental requirement for divers learning enhancing techniques. On this basis we will present a series of recent findings concerning the targeted reactivation of foreign vocabulary by auditory cues during sleep, its impact on vocabulary learning and associated neural activity. Finally, we propose a working model on how oscillatory activity during sleep supports reactivation, stabilisation and integration of newly learned words. We conclude that enhancing language learning during sleep by targeted memory reactivation (TMR) provides an exciting new investigation tool, stimulating future research to further understand neural mechanisms underlying sleep-benefits in language acquisition and consolidation.

\section{Memory and sleep}

Language learning is closely tied to basic processes of memory, which can be broadly 
divided into three core stages: encoding, consolidation and retrieval (Gabrieli, 1998). During encoding, new and initially labile memory traces are formed that are still highly susceptible to interference. During subsequent memory consolidation, these newly encoded memories are stabilised and strengthened. In addition, the new memory representations are gradually integrated into pre-existing knowledge networks on the cortical level for long-term storage. Finally during retrieval, memories are assessed and recalled.

While encoding and retrieval are clearly tied to the state of wakefulness, sleep plays a crucial role in the consolidation of newly encoded memories (see Rasch and Born, 2013, for a comprehensive review): Sleep after learning benefits memory consolidation, leading to stronger and better integrated memory traces after sleep, supporting improved retrieval performance. These beneficial effects of sleep on memory have been consistently demonstrated for diverse types of memories (Walker \& Stickgold, 2004), ranging from priming, conditioning and procedural motor memories (Menz et al., 2013; Plihal \& Born, 1999; Plihal \& Born, 1995) to declarative episodic memories including vocabulary learning (Gais \& Born, 2004; Gais, Lucas, \& Born, 2006). Furthermore, sleep has been demonstrated to support processes of abstraction, inference, and insight (Ellenbogen, Hu, Payne, Titone, \& Walker, 2007; Lewis \& Durrant, 2011; Wagner, Gais, Haider, Verleger, \& Born, 2004). First concepts were formulated almost a century ago and assumed that sleep might solely protect memories passively by a reduction of external interference after learning (Jenkins \& Dallenbach, 1924). Since then, substantial evidence has accumulated indicating that the memory function of sleep goes far beyond these early assumptions by providing consistent support for an active role of sleep for the consolidation of memories (e.g. Gais et al., 2006; Born and Wilhelm, 2012; Walker and Stickgold, 2004). According to the theoretical account of active system consolidation during sleep (Diekelmann \& Born, 2010), which we will describe in more detail below, a central aspect of the active role of sleep on memory consolidation is the notion that newly encoded memories are spontaneously and repeatedly reactivated during sleep. These reactivations are thought to stabilize and strengthen newly encoded memories for the long-term and to integrate them into pre-existing knowledge 
networks. Similarly, according to the recent version of the synaptic homeostasis hypothesis "synapses activated strongly and consistently during sleep survive mostly unchanged and may actually consolidate” (Tononi \& Cirelli, 2014, page 15).

\section{Memories are spontaneously reactivated during sleep}

Supporting the notion of a critical role of reactivations during sleep for memory consolidation, replay activity during sleep has been consistently reported in memory-related brain structures, particularly in the hippocampus, in rodents as well as in humans (O’Neill, Pleydell-Bouverie, Dupret, \& Csicsvari, 2010; Pavlides \& Winson, 1989; Peigneux et al., 2004; Peyrache, Khamassi, Benchenane, Wiener, \& Battaglia, 2009). Pioneering rodent studies could demonstrate that the spatial and even the temporal pattern of neuronal firing, occurring during exploration of a novel environment, are spontaneously reactivated in the same order during subsequent Non-rapid eye movement (NREM) sleep (Skaggs \& McNaughton, 1996; Wilson \& McNaughton, 1994). Succeeding experiments additionally indicated that spontaneous memory reactivations are typically time-compressed by a factor of 10-20 (O’Neill et al., 2010) and are closely related to specific oscillations in the hippocampus, the so called hippocampal sharp-wave ripples (SW-R, large amplitude sharp wave followed by a high frequency oscillation in the $200 \mathrm{~Hz}$ range) (Ylinen et al., 1995). Replay activity during sleep has not only been found in the hippocampus but as well in various other memory related brain regions (i.e., prefrontal cortex, ventral striatum etc. (Ji \& Wilson, 2007; Pennartz et al., 2004; Peyrache et al., 2009)), possibly mirroring the gradual redistribution and integration of memory representations in cortical brain structures.

Based on these findings, the active system consolidation theory postulates that the covert and repeated reactivations of newly encoded memories during NREM sleep sub-serve these system consolidation processes of reorganization and integration (Diekelmann \& Born, 2010; Stickgold \& Walker, 2013). System consolidation refers to the two-stage model of memory consolidation (Marr, 1971; McClelland, McNaughton, \& O’Reilly, 1995), stating 
that initial encoding of memories critically relies on a fast learning memory system (i.e., the hippocampus for declarative memories). After encoding, the information is gradually redistributed into a second, slow-learning memory system in cortical brain areas and integrated into pre-existing knowledge networks. Thereby, memory representations gradually loose their retrieval-dependency on hippocampal structures. According to the active system consolidation theory, this process of gradual system consolidation is actively supported by sleep based on repeated memory reactivations interleaved with reactivation of associated older memories (Rasch \& Born, 2007).

It is further assumed that the reactivation and redistribution of memory representation during post-learning sleep is closely linked to sleep-specific oscillatory brain signals characterising NREM sleep: neocortical slow oscillations, thalamo-cortical spindles and hippocampal SW-Rs (Diekelmann \& Born, 2010). The slow $(<1 \mathrm{~Hz})$ oscillation is primarily generated in the neocortex (Steriade, 2006; Timofeev \& Chauvette, 2011). It spreads across the neocortex and mirrors global synchronous changes of neural activity alternating between periods of increased excitability (depolarized "up-states") and neuronal quiescence (hyperpolarized "down-state"). Furthermore, slow oscillations affect activity in other brain structures, such as the striatum, locus coeruleus and the hippocampus, potentially coordinating communication between these brain regions and the neocortex (Genzel, Kroes, Dresler, \& Battaglia, 2014). During NREM sleep stage 2, single slow oscillations might occur as K-complexes which are internally generated but can also be triggered by sensory stimulation during sleep (Colrain, 2005).The active system consolidation theory ascribes the slow oscillation the role of a time giving pace maker: The depolarizing up-states of the slow oscillation drive repeated reactivations of memory representations in the hippocampus (associated with hippocampal SW-Rs) together with thalamo-cortical sleep spindles (waxing and waning oscillatory events in the sleep EEG in the 11-15 Hz frequency range), thereby enabling the formation of spindle-ripple events. Via increasing $\mathrm{Ca}^{2+}$ influx into neocortical pyramidal cells, these spindle-ripple events are assumed to provide an important basis for the 
information redistribution by which reactivated hippocampal memory information trigger, enduring plastic changes in neocortical areas (Born \& Wilhelm, 2012).

In sum, the theory postulates a crucial role of slow oscillatory activity in synchronizing hippocampal memory reactivations with thalamo-cortical spindle activity, thereby redistributing and integrating the memories into neocortical long-term stores (Bergmann, Mölle, Diedrichs, Born, \& Siebner, 2012; Dongen, Thielen, Takashima, \& Barth, 2012; Rasch \& Born, 2013; Rihm, Diekelmann, Born, \& Rasch, 2014; Ritter, Strick, Bos, Van Baaren, \& Dijksterhuis, 2012). How can we empirically test the theoretical assumption of a critical role of memory reactivations during sleep for processes of memory consolidation? If reactivation during sleep indeed plays a functional role for the consolidation of memories, experimentally increasing these reactivations should have beneficial consequences for consolidation processes during sleep and result in improved memory performance the next day. Before we will present evidence for the beneficial effect of TMR by re-exposure to memory cues during sleep, we will briefly discuss the capability of the sleeping brain to processes sensory stimuli and discriminate semantic categories.

\section{Sensory processing and semantic discrimination during sleep}

Traditionally the sleeping brain has been conceived as shut down and quiescent, given that sleep is associated with a reversible reduction in behavioral responsiveness. Processing of external sensory information was believed to be generally reduced or even impossible due a reduction of information flow via the thalamus ("thalamic gating") (McCormick \& Bal, 1994; Mircea Steriade \& Timofeev, 2003) Thalamic gating is mainly due to massive hyperpolarization of thalamo-cortical neurons during sleep originating from brainstem mechanisms (McCormick \& Bal, 1994). These processes result in a firing of widespread neuron populations in a burst mode, which blocks a major part of the incoming sensory information at the thalamic level (Coenen \& Vendrik, 1972). Importantly this statedependent gating occurs as well in the central olfactory pathway that lacks a thalamic relay. 
Here the gating originates in the brainstem ascending reticular formation, in synchrony with the thalamic gating (Murakami, Kashiwadani, Kirino, \& Mori, 2005).

However, recent findings clearly show that neurons of the primary auditory system are still responsive during sleep (Edeline, Dutrieux, Manunta, \& Hennevin, 2001; Issa \& Wang, 2008; Nir, Vyazovskiy, Cirelli, Banks, \& Tononi, 2015; Pena, Perez-Perera, Bouvier, \& Velluti, 1999), suggesting that the thalamus might not be as effective in gating sensory information as previously believed. Astonishingly, in a study by Issa \& Wang (2008) neurons in the primary auditory cortex as well in the secondary auditory cortical areas displayed on average even similar discharge patterns during sleep as compared to wakefulness. With regards to olfactory processing, cortical odor-evoked activity is reduced with regards to the wake state but not eliminated during SWS (Wilson \& Sullivan, 2011). Accordingly, the sleeping brain responds to external input, at least to some extent.

Along similar lines, several human studies measuring event-related potentials (ERPs) during sleep have repeatedly demonstrated that the sleeping brain is able to discriminate deviant auditory tones from repetitive ones during all sleep stages (for a review see, (Bastuji \& García-Larrea, 1999)). Specifically during NREM sleep stage 2 and SWS, deviant tones elicit K-complexes / EEG surface slow waves of higher amplitude as compared to the standard tone. Using functional magnetic resonance imaging (fMRI), Dang-Vu and colleagues (2011) showed that tones played during NREM sleep activate the primary auditory cortex and thalamus, as well as some regions involved in the processing of auditory stimulation (cerebellum, middle frontal gyrus, precuneus and posterior cingulate gyrus). The activations were particularly strong when the tones elicited a K-complex, suggesting that induced single slow waves after an auditory stimulus might be indicative for enhanced auditory processing at the cortical level, which is in line with earlier findings (Czisch et al., 2009). Interestingly, this activity pattern induced by presenting the tone during NREM sleep was completely absent when the tone was played during the occurrence of a sleep spindle. The authors concluded that sleep spindles can strongly reduce the transmission of sensory information to the cortex and might transiently isolate the cortex from auditory input. 
In addition to the detection of physical stimulus characteristics, the ability to discriminate semantic meaning of words is also preserved during sleep. Presenting one's own name during NREM sleep elicits increased responses in early portions of K-Komplexes and leads to a stronger activation of auditory brain areas as compared to the presentation of other names or tones (Perrin, García-Larrea, Mauguière, \& Bastuji, 1999; Portas et al., 2000; Pratt, Berlad, \& Lavie, 1999). Furthermore, presentation of semantically incongruent word pairs during sleep led to an enhanced N400, which is typically elicited through the perception of semantically unrelated information during wakefulness and is understood as marker of verbal discordance (Brualla, Romero, Serrano, \& Valdizán, 1998; Perrin, Bastuji, \& Garcia-Larrea, 2002). An increased N400 was even observed after presentation of incongruent sentences during N2 sleep (Ibáñez, López, \& Cornejo, 2006), strongly suggesting that semantic discrimination is preserved during NREM sleep to some extent. In sum, the sleeping brain is clearly able to process auditory and olfactory information, to establish short-lasting representation of sensory memory and to discriminate sensory input depending on its semantic content. This capability of the sleeping brain to successfully process external sensory information provides an important prerequisite for inducing memory reactivations during sleep by associated memory cues, which we will discuss in the next section.

\section{Inducing reactivations by cueing during sleep}

According to the concept of context-dependent memory (Godden and Baddelley, 1975; Smith and Vela, 2001), the learning context is always associated with the to-be-learned information and becomes integrated in the stored episodic memory trace. Thus, later reexposure to the learning context reactivates the memory and facilitates voluntary retrieval. In a first attempt to prove the functional significance of reactivations for memory formation, we used contextual odors to reactivate memories during sleep (Rasch, Büchel, Gais, \& Born, 2007). Olfactory stimuli have a high potency as contextual retrieval cues (Chu \& Downes, 2002) and can be presented during sleep without disturbing on-going sleep (Carskadon \& 
Herz, 2004). In our study, participants learned an object-location task while smelling a rose odor. During subsequent SWS, either the odor was administered again to the participants or an odourless vehicle substance was used, in a within-subject design. In support for the critical role of reactivations for memory consolidation during sleep, odor re-exposure during NREM sleep significantly enhanced recall performance the next day as compared to nights without the odor. Furthermore, re-exposure to the odor during SWS activated the hippocampus as revealed by fMRI (Rasch et al., 2007, Rasch \& Born 2007). Thus, re-exposure to the contextual odor during sleep triggered hippocampal reactivations of the learning material, which then benefited the consolidation, strengthening and integration of the newly encoded memories into long-term knowledge networks. Importantly, we have now successfully replicated the beneficial effect of odor re-exposure during NREM sleep on memory consolidation in two additional and independent studies (Diekelmann, Büchel, Born, \& Rasch, 2011; Rihm et al., 2014;). Furthermore, Hauner and colleagues (2013) reported that reexposure to odors during a nap reduces fear memories in humans. In contrast, inducing "Eodors" during sleep in rodents by electrical activation of neurons within the piriform cortex strengthened associated fear-memories (Barnes \& Wilson, 2014). Nonetheless, differences in the conditioning procedure and the experimental protocol between those studies might be responsible for the contradictory results (for a detailed discussion see Diekelmann \& Born, 2015). Still, in opposition to reactivation during NREM sleep, we do not observe any memory benefits of odor re-exposure during REM sleep even after controlling for the time interval of odor re-exposure during sleep with respect to the learning phase (Cordi, Diekelmann, Born, \& Rasch, 2014; Rasch et al., 2007).

In addition to odors, several studies were able to demonstrate that also auditory stimuli presented as memory cues during NREM sleep effectively improve consolidation processes during sleep (Oudiette \& Paller, 2013 for a review). For example, individual sounds indicative for a certain to-be-learned card location activate parahippocampal regions during sleep and improve subsequent memory retrieval (Dongen et al., 2012; Rudoy, Voss, Westerberg, \& Paller, 2009). Furthermore, auditory cueing during sleep activates 
hippocampal neurons (Bendor \& Wilson, 2012), enhances skill learning (Antony, Gobel, O’Hare, Reber, \& Paller, 2012; Schönauer, Geisler, \& Gais, 2013), promotes explicit knowledge of a motor task (Cousins, El-Deredy, Parkes, Hennies, \& Lewis, 2014) extinguishes fear memories (He, Sun, Li, Zhang, \& Shi, 2015; Rihm \& Rasch, 2015; Rolls et al., 2013), enhances the consolidation of emotional memories (Cairney, Durrant, Hulleman, \& Lewis, 2014) and might even affect building of stereotype (Hu et al., 2015). Importantly, such beneficial effects of cueing during sleep depends on the structural integrity of the hippocampus, as these effects are completely absent in patients with unilateral or bilateral hippocampal sclerosis (Fuentemilla et al., 2013). Furthermore, they depend on the prior performance level in healthy subjects (Creery, Oudiette, Antony, \& Paller, 2015). Thus, TMR during sleep activates hippocampal regions and benefits memory consolidation processes of newly encoded stimuli, resulting in better long-term memory. In the next paragraph we will turn to language learning and sleep, and how we can use similar approaches and techniques discussed so far to improve vocabulary learning or other aspects of language learning.

\section{Sleep and language learning}

Language learning mainly encompasses the association of symbols and sounds with meaningful concepts and allows us to describe the external environment and our internal thoughts (Pinker, 2000). As sleep is clearly involved in consolidation processes underlying the formation, strengthening and integration of associations (see above), it appears quite obvious that sleep may play a fundamental role in language learning. In support for this notion, numerous findings provide evidence for the beneficial influence of sleep on establishing the "mental lexicon", particularly with respect to word learning. For example, sleep has been proven to support vocabulary learning in children and adolescents (Gais et al., 2006; Henderson et al., 2012), to promote the generalization of word meaning in infants (Friedrich, Wilhelm, Born, \& Friederici, 2015), to benefit speech production (Gaskell et al., 2014), speech recognition (Fenn, Nusbaum, \& Margoliash, 2003) and importantly the integration of new words into pre-existing knowledge networks (Dumay \& Gaskell, 2007). 
Interestingly, important oscillatory indicators of this integration processes were sleep spindles during the retention interval (Tamminen, Payne, Stickgold, Wamsley, \& Gaskell, 2010) and theta activity during later recognition testing (Bakker, Takashima, Hell, Janzen, \& McQueen, 2015).

While most studies linking language learning and the memory function of sleep concentrated on learning and integration of new words, some recent evidence suggests that sleep also supports grammar learning. Three recent studies could demonstrate that sleep facilitates the learning and extraction of new grammatical rules: Specifically, infants who were allowed to sleep after exposure to an artificial grammar demonstrated greater rule abstraction (Gómez, Bootzin, \& Nadel, 2006). In the same vein in adults, sleep enhances rule abstraction to an artificial grammar (Nieuwenhuis, Folia, Forkstam, Jensen, \& Petersson, 2013) and the learning of hidden linguistic rules (Batterink et al., 2014). In this context, the known beneficial effects of sleep for generalization and abstraction seem to be specifically important (e.g. Stickgold \& Walker, 2013). Furthermore, there is first evidence that grammatical generalization can as well be influenced by TMR during sleep (Batterink \& Paller, 2015). As in prior studies investigating sleep and grammar learning, participants learned the grammatical rules of an artificial language in this study. Importantly, phrases from the previously learned language were repeatedly presented during a subsequent afternoon nap. As compared to participants from a control condition, which received stimuli from an unrelated task during sleep, participants re-exposed to the phrases during sleep showed enhanced grammatical generalization afterwards, indicating that grammar learning may profit from cueing during sleep.

From a theoretical perspective, Davis and Gaskell (2009) proposed a model for word learning named the Distributed Cohort Model, which is closely linked to the active system consolidation theory of memory formation during sleep described above. The model distinguishes between two stages of learning and lexicalization of new words: At a first stage the hippocampus is pivotal and the initial familiarization with the new word leads to the formation of a sparse individual memory representation of the new word. These newly 
encoded memory representations are then replayed off-line, particularly during sleep, resulting in a creation and strengthening of a more distributed and integrated lexical representation (i.e.: phonological form and meaning of spoken words) in neocortical longterm memory. During this second stage, the distributed lexical representation is allowed to compete with overlapping representations during speech perception (Davis \& Gaskell, 2009), enabling automaticity in word recognition. In animal models of language learning, reactivation during sleep might similarly be critical for song learning in birds (Dave \& Marholiash, 2000), although the general mechanisms of system consolidation during sleep may differ between mammals and birds (Rattenborg, Martinez-Gonzalez, Roth, \& Pravosudov, 2011). Thus, reactivation processes during sleep may be the key for an efficient consolidation processes in word learning or even language learning in general.

\section{Improving vocabulary learning by cueing during sleep}

If reactivation is indeed critical for language learning during sleep, TMR during sleep should lead to significant improvements in language learning. In a recent study we made the first important step in this direction. We tested whether re-exposure to complex verbal cues during sleep induces reactivations during sleep and improves vocabulary learning (Schreiner \& Rasch, 2015a). In our study, participants learned new Dutch words and their German translations in the evening. During subsequent NREM sleep, we replayed only the prior learned Dutch words (without the German translation) to reactivate the newly learned association between the Dutch and German words. During post-learning sleep, only half of the newly learned words were replayed (cued words) while the others were not (uncued words, see Figure 1A, for a summary of the experimental procedure). Consistent with the theoretical predictions, replay of the Dutch words during sleep led to improved memory recall for the German translation of the cued words tested after sleep as compared to the uncued words (see Figure 1B). Interestingly, recall performance of the uncued words was quite similar to an additional group receiving no verbal cues during sleep, indicating that the benefits of auditory cueing indeed exceed the normal consolidation effects of sleep on 
memory and that cueing during sleep on memory occurred without any obvious "costs" (i.e. verbal cueing seems not have disturbed ongoing consolidation processes during sleep). In addition, we showed in a total of four independent experimental groups that the beneficial effect of post-learning cueing for vocabulary learning is specific to sleep: When participants learned the Dutch-German word-pairs in the evening and listened to the Dutch cues while being awake during night-time, retrieval performance for cued and uncued words did not differ in spite of an identical circadian timing of learning, retention and retrieval phases (Figure 1C). To exclude tiredness as a confounding factor, we also tested the effect of vocabulary cueing during active and passive daytime wakefulness (Schreiner \& Rasch, 2015b). Again, no benefits of cueing emerged although participants were well rested during retrieval testing (see Figure 1D). Independent of cueing, the results replicate the general benefits of sleep as compared to wakefulness for vocabulary learning, which can be further improved by cueing during sleep.

Note that in all of these studies we have only used the Dutch word without the German translation as memory cues. This reactivation procedure is based on the idea of reactivating the German translation during sleep by presenting solely the memory cue (the Dutch word). However, it is conceivable that the presentation of the whole word-pair during sleep could potentially be even more beneficial for memory: Re-exposure to the Dutch Word immediately followed by its German translation might reactivate and strengthen the whole memory traces to a greater extent as compared to re-exposure to the Dutch word alone. To test this important question, in a subsequent study (Schreiner, Lehmann \& Rasch, 2015) participants learned again Dutch words and their German translations in the evening. During subsequent sleep, one third of the newly learned words were replayed without the German translation (single cue), one third was replayed immediately followed by the correct German translation (cue + feedback) and one third was not replayed during sleep (uncued). While we could replicate our former finding that cueing of single Dutch words enhances memory performance, the benefits of cueing Dutch vocabulary during sleep were completely blocked when memory cues were directly followed by correct feedback (Figure 1E). In an additional group, exactly the same 
result pattern emerged when the Dutch word was immediately followed by a false German translation, without any further impairment in the feedback condition. This result pattern strongly suggested that the blocking of the benefits of single word cueing during sleep does not depend on the content of the second word but more crucially on the timing of the second stimulus (Figure 1F). We substantiated this conclusion by running a third group varying the timing and the content of the second auditory stimulus. Here, replayed Dutch cues were again followed by the correct German translation but with a longer inter-stimulus interval $(1,500 \mathrm{~ms}$ instead of $200 \mathrm{~ms}$, cue + late feedback), while others were immediately followed by a pure tone (cue + tone). We hypothesized, that if the timing of the feedback is substantial and further the content does not play any crucial role, a neutral second stimulus such as a tone should as well block the beneficial effects of cueing, while a longer inter-stimulus interval might resolve that. Additionally, one third of the Dutch group was again not replayed during sleep (uncued). Importantly, while playing a tone immediately after the Dutch word completely blocked any benefits, the memory enhancing effect of cueing during sleep reemerged when feedback was played after an additional time delay (Figure 1G). The result pattern clearly indicates that a undisturbed period of approximately 1-2 seconds after cue presentation during sleep is critically needed, in order to let reactivation-induced plasticity processes unfold their benefits on the stabilisation of memory representation of newly learned foreign vocabulary.

Interestingly, presenting the whole memory content (i.e. 'cue + correct feedback') did not further strengthen memories during sleep in our study, even when the feedback was presented with a sufficient time delay. Several reasons might explain this finding. First, there might be a restricted capacity for reactivation processes during sleep. Evidence for such a limited capacity comes from a rodent study accomplished by Bendor \& Wilson (2012), in which cueing did not increase the overall number of reactivation events on the level of the hippocampus. Second, there might be a ceiling effect on the behavioral memory benefit of cueing: As cues were repeatedly presented during sleep, additional presentation of the German word might have triggered reactivation without additionally enhancing memory 
strength. And third, directionality of cueing during sleep is still unclear. As words were acquired with a clear direction during the learning phase (Dutch -> German), it is unclear whether presentation of the German word alone results in behavioral memory benefits. Finally, repeated auditory cueing might have provoked refractoriness in slow oscillation and sleep spindle generating networks. Recent studies demonstrated such a refractoriness with regards to the presentation of consecutive click sounds during sleep (Ngo, Claussen, Born, \& Mölle, 2013; Ngo et al., 2015).

\section{Oscillatory correlates of vocabulary cueing during sleep}

During wakefulness, a cascade of molecular mechanisms is necessary to stabilise memories immediately after their encoding or reactivation, as described in studies concerning consolidation or reconsolidation processes (McGaugh, 2000; Nader \& Hardt, 2009; Tronson \& Taylor, 2007). In contrast, plastic processes on the molecular level underlying stabilisation after reactivation during sleep are still largely unknown, but might possibly involve similar mechanisms (Havekes, Meerlo, \& Abel, 2015). So far, oscillatory parameters associated with successful memory reactivation during sleep have provided first insights into plasticity processes supporting stabilisation, strengthening and integration after reactivation during sleep.

During wakefulness, oscillatory activity in the theta $(4-7 \mathrm{~Hz})$ and gamma $(25-100 \mathrm{~Hz})$ range have been consistently linked to the successful encoding and retrieval of new declarative information (Nyhus \& Curran, 2010). Theta as well as gamma activity are associated with processes of long term potentiation (LTP) and synaptic plasticity, thereby facilitating the encoding of new memories (Axmacher, Mormann, Fernández, Elger, \& Fell, 2006; Huerta \& Lisman, 1995; Hyman, Wyble, Goyal, Rossi, \& Hasselmo, 2003). Furthermore, theta-gamma interactions seem to provide the means for the temporal ordering and binding of individual memory representations. Gamma rhythms are assumed to represent and bind the perceptual features of a memory representation during encoding and to supply a coherent pattern of activity as input to the hippocampus, while theta activity provides the mechanism to order 
those representations (Lisman \& Jensen, 2013; Nyhus \& Curran, 2010). With regards to the retrieval of declarative memories, those theta and gamma patterns are assumed to cause the reinstatement of the entire memory representation in the cortex through hippocampal-cortical feedback projections (Nyhus \& Curran, 2010). Theta activity also reflects the successful integration of new words in the existing mental lexicon (Bakker et al., 2015) and the internal strength of episodic memory traces (Klimesch et al., 2006). For example, theta and gamma activity is typically increased for correctly recognized words (hits) as compared to correctly rejected new words (Mormann et al., 2005; Osipova et al., 2006; Trimper, Stefanescu, \& Manns, 2014). In our recent work (Schreiner, Göldi, \& Rasch, 2015) we similarly observed increased theta activity for correctly recognized Dutch vocabulary during recognition testing after sleep. Furthermore, correct recognition of words that had been replayed during sleep elicited stronger theta activity compared to uncued words (Figure 2), possibly reflecting an enhanced integration of the newly learned words into mental lexicon by cueing during sleep (Bakker et al., 2015) and the presence of a stronger memory trace (Klimesch et al., 2006). In parallel to these findings from waking studies, we observed also strong evidence during NREM sleep for increased theta activity after successful cueing as compared to unsuccessful cueing. In our first study using solely single cues (Schreiner \& Rasch, 2015a), a cueingrelated increase in induced theta power during NREM sleep was clearly visible and strongest for those words that were gained by cueing during sleep (i.e., not remembered before sleep, but successfully retrieved after cueing during sleep). In addition, we observed an increase in oscillatory activity in the sleep spindle range specifically during SWS. Similarly in our subsequent sleep study (Schreiner, Lehmann, \& Rasch, 2015), presenting single cues (i.e., the Dutch word alone) during NREM sleep consistently resulted in an increase in oscillatory theta and spindle activity during trials of successful memory reactivation. However, when the correct or false German translation was presented immediately following the first memory cue, this increase in theta and spindle oscillations were completely blocked. The blockade of this important oscillatory pattern was also observed when presenting the pure tone after the cue. In all of these three conditions, abolishing oscillatory activity on the neural level was 
paralleled by a blockade of memory benefits of cueing on the behavioural level. In contrast, the increase in theta and spindle oscillation re-emerged when the verbal feedback was presented after the time delay of $1500 \mathrm{~ms}$, together with the behavioural memory benefit of cueing during sleep. Still, an open question with regards to these empirical findings is, which processes constitute such critical time frames after cueing during sleep. In line with the active system consolidation theory one might speculate that the benefits of cueing depend on the timing of slow oscillations / K-complexes. Slow oscillations coordinate reactivationassociated SW-R activity (Isomura et al., 2006; Sirota \& Buzsáki, 2005; Sirota, Csicsvari, Buhl, \& Buzsáki, 2003), with SW-Rs occurring mostly at the transitions between down and up states (Battaglia, Sutherland, \& McNaughton, 2004; Peyrache, Battaglia, \& Destexhe, 2011). Thus, slow oscillations should as well constitute a crucial key factor for cueing success by providing the temporal frame for reactivation processes and the dialogue between the neocortex and subcortical structures. Conforming with this assumption the number of slow waves following the memory cue was similarly associated with cueing success in all of our studies. With regards to the observed critical time window of 1- 2 seconds which seems to be needed in order to let cueing specific effects unfold, one might assume that at least one full slow-oscillation (including one down and one up-state) would be required after cueing to improve memory strength, as the frequency range of slow waves ranging from 0.5 to $1.5 \mathrm{~Hz}$ exactly encompasses this time range ( 0.75 - 2 seconds). Speaking for such an interpretation, unpublished work suggests that replay activity after cueing occurs mostly during the slow oscillations up-states after cue presentation (Antony, Piloto, Paller, \& Norman). Additionally, there is strong evidence that neocortical slow oscillation induce grouping effects on spindle and ripple activity, with synchronized activity during the slow oscillations up-states (Clemens et al., 2007; Staresina et al., 2015). Given the pattern of results obtained in our studies we would assume that slow oscillations have a similar time-giving effect on theta activity, with theta activity being coupled to the slow oscillations up-states. Taken together, after cueing during sleep, the occurrence of a synchronized up-state and its associated oscillatory processes (endogenous reactivation signals, SW-R, spindles / theta oscillations and associated 
plastic processes, e.g. early gene expression, calcium influx etc.) are possibly required to allow memory benefits to unfold. Therefor, immediate presentation of a second word right after the cue might disturb the development of a synchronized up-state and block the memory benefits of cueing.

Thus overall, the behavioural memory benefits of cueing vocabulary during sleep are tightly linked to activity increases in theta and spindle oscillations. This result pattern provides strong evidence for a critical role of oscillatory processes in the theta and spindle range and/or its associated molecular plastic processes together with the occurrence of slow oscillations for a successful stabilisation of reactivated memories traces during NREM sleep.

\section{A working model for stabilising reactivated memories during sleep}

Unlike sleep spindles and slow oscillations, theta activity is not yet included in current theoretical models of sleep and memory processes during NREM sleep. However, several recent findings support the notion that theta activity during NREM sleep might be implicated in on-going memory processing during sleep. For example, faster theta frequency and increased theta power during NREM sleep predicted better subsequent memory performance in patients with Alzheimer's disease and amnestic mild cognitive impairment, as well as healthy participants (Hot et al., 2011; Schabus, Hoedlmoser, Pecherstorfer, \& Kloesch, 2005; Westerberg et al., 2012). In addition, theta activity is increased during processing of rare stimuli in a mismatch paradigm, suggesting an involvement of theta oscillations during sleep in sensory memory processing of auditory stimuli (Karakaş, Cakmak, Bekçi, \& Aydin, 2007). Furthermore, a recent study demonstrated that spike timing during delta-nested theta rhythms controls a reciprocal interaction between deep and superficial cortical layers mimicking the alternating cortical dynamics of sensory and memory processing during wakefulness (Carracedo et al., 2013). While our findings additionally suggest that theta oscillations may play a crucial role in association with reactivation and stabilisation processes during sleep, the exact contribution of theta oscillations for consolidating memories during sleep and its potential interplay with sleep spindles and slow oscillations is still unclear. 
In our working model (Figure 3), we propose that theta activity represents the successful reinstatement of the memory representation after cueing. Thus, increase in theta activity will occur when (a) the association between the memory cue and the to-be-reactivated memory representation is strong enough and (b) when the cue is presented at an appropriate time and in an appropriate way (i.e. intensity etc.) to successfully reinstate the associated memory representation. We assume that increases in theta activity are in principle independent of the macro state of the brain (i.e. sleep vs. wakefulness, NREM vs. REM sleep etc.). Thus, increases in theta activity after successful memory reactivation should occur during NREM sleep, waking and maybe even REM sleep. As described above, increases in theta activity have been consistently observed during successful retrieval and recognition during wakefulness (Jacobs, Hwang, Curran, \& Kahana, 2006; Klimesch et al., 2001) as well as during successful reactivation during NREM sleep (Schreiner \& Rasch, 2015a) and is assumed to coordinate replay activity during working memory maintenance (Fuentemilla, Penny, Cashdollar, Bunzeck, \& Düzel, 2010).

Based on findings from waking studies (Nyhus \& Curran, 2010), we similarly assume that oscillatory theta and gamma during NREM cause the hippocampus-dependent reinstatement of entire memory representations in the cortex. In addition, theta-frequency activity might facilitate the initiation of synaptic potentiation (Larson, Wong, \& Lynch, 1986) and might play a role in coordinating the activity of the hippocampus and the prefrontal cortex (Benchenane et al., 2010). However in our view, increases in theta activity do not necessarily predict the fate of the reactivated memory representation, as the latter depends mainly on the macro state of the brain together with subsequent external input: During waking, reactivated memories might either be strengthened (if correct feedback is given or when they are actively re-encoded), unchanged or even destabilized (if interfering input occurs after reactivation according to reconsolidation theory (Nader \& Hardt, 2009)). In contrast during NREM sleep, successfully reactivated memories are always strengthened as long as stabilization processes occurring immediately after reactivation remain undisturbed.

Concerning sleep spindles, we assume that sleep spindle oscillations occurring in parallel or 
shortly after increases in theta activity are necessary to support the stabilization, strengthening and integration after successful reinstatement of the memory representation by the memory cue. The active system consolidation hypothesis assigns sleep spindles a critical role in redistributing reactivated hippocampal memories to neocortical circuits (Born \& Wilhelm, 2012): Hippocampal reactivation signals are nested in individual spindle troughs (Mölle, Eschenko, Gais, Sara, \& Born, 2009; Staresina et al., 2015; Siapas \& Wilson, 1998; Sirota \& Buzsáki, 2005) and sleep spindles prime and/or maintain long-term potentiation (LTP) in cortical networks by triggering $\mathrm{Ca}^{2+}$ inflow for subsequent synaptic plastic processes (Contreras, Destexhe, \& Steriade, 1997). However, sleep spindles per se are not necessarily directly associated with hippocampal activity (Andrade et al., 2011), and reactivation processes in rodents occur slightly before the appearance of spindles (Peyrache et al., 2009). In addition, the cortex is presumably even deafferented from hippocampal and thalamic inputs during sleep spindles (Peyrache et al., 2011), possibly indicating that sleep spindle activity might not be directly associated with the information redistribution but rather stabilizes and strengthens memory traces, which were reactivated just before the sleep spindle (Genzel et al., 2014).

In our view, spindle activity enables local, undisturbed cortical reprocessing of previously reactivated memories, while the reactivation itself is associated with theta activity. Thus, a successful reinstatement of memory representation (as indicated by transient increases in theta activity) together with subsequent occurrence of oscillatory spindle activity might be necessary to induce plastic changes underlying the stabilization of memories upon their reactivation during sleep. Disturbing these combined oscillatory signals (e.g., by presenting auditory stimuli immediately after successful reactivation) disrupts the reinstatement (theta) and stabilization (spindle) process and blocks the beneficial effect of cueing during sleep (see Figure 1E-G). Our working model would also predict that successful memory reactivation during REM sleep could result in increases in theta activity (i.e. successful memory reinstatement), but does not lead to a stabilization of the memory trace due to missing spindle oscillations. Conversely (if at all possible), inducing spindle activation immediately after 
reactivation during REM sleep should result in memory benefits of cueing.

Still, as we derived our working model from experimental data where memory reactivations were externally induced by cueing procedures, one might ask whether the same oscillatory patterns would be traceable when it comes to spontaneous reactivation processes. In order to clarify this important issue, future studies would need to search for the co-occurrence of oscillatory activity in theta and spindle range and correlate these measures with subsequent memory performance.

What role do slow oscillations play in our working model? According to the active system consolidation theory, slow oscillations represent a time-giving pace maker, orchestrating neural processes related to the reactivation of prior encoded memories during sleep. Slow oscillations have been repeatedly shown to group sleep spindles (Mölle, Bergmann, Marshall, \& Born, 2011; Mölle, Marshall, Gais, \& Born, 2002) whereas a recent report indicated theta to be nested in ongoing delta activity (Carracedo et al., 2013). Furthermore, several lines of research suggest that slow oscillations effectively coordinate SW-R activity in the hippocampus (Isomura et al., 2006; Sirota \& Buzsáki, 2005; Sirota et al., 2003) and thus potentially also hippocampal reactivation processes. While successfully reactivated and strengthened memories in our studies were more often followed by EEG surface slow waves, words that did not benefit from cueing during sleep were also followed by slow oscillations. Similarly, pharmacological enhancement of slow wave activity alone without increasing spindle activity does not improve overnight memory consolidation (Feld et al., 2013). IN addition, benzodiazepines are known to enhance sleep spindle activity and suppress slow wave activity (Arbon, Knurowska, \& Dijk, 2015; Brunner, Dijk, Münch, \& Borbély, 1991). However, the effects of benzodiazepine administration on memory consolidation during sleep are quite inconsistent, including detrimental effects (Hall-Porter, Schweitzer, Eisenstein, Ahmed, \& Walsh, 2014), no effects (Melendez et al., 2005) as well as positive effects (Kaestner, Wixted, \& Mednick, 2013; Mednick et al., 2013). Thus, neither enhancing slow wave nor spindle activity alone is sufficient to enhance the memory function of sleep, while simultaneously increasing slow wave and spindle activity by auditory or electric stimulation 
does (Marshall, Helgadóttir, Mölle, \& Born, 2006; Hong-Viet V Ngo, Martinetz, Born, \& Mölle, 2013; H.-V. V. Ngo et al., 2015). Thus, slow oscillations alone are not effective alone, but might represent a relevant pre-requisite for cueing success by providing the temporal frame for reactivation processes and the dialogue between the neocortex and subcortical structures. Within this time frame, activity in the theta and spindle range are then critical for successful memory reinstatement and its subsequent stabilization / strengthening after cueing during sleep. Note that we did not synchronize the verbal cues to a specific phase of endogenous EEG slow waves. Thus, it remains an intriguing question, whether replaying the auditory cues precisely into the slow oscillations up-states, which are associated with enhanced neural activity and might represent brief wake like fragments during sleep (Destexhe, Hughes, Rudolph, \& Crunelli, 2007), would further enhance the beneficial effects of cueing vocabulary during sleep.

\section{Conclusion}

In sum, the findings reviewed above demonstrate the crucial role of sleep in language and specifically word learning. It has been shown that sleep promotes divers aspects of language learning, from word learning to the abstraction of grammar rules (Batterink et al., 2014; Henderson et al., 2012) and possibly constitutes an ideal state in order to facilitate and accelerate distinct learning processes. In this vein, evidence that foreign vocabulary are capable of inducing such reactivation processes and thereby enhance subsequent memory performance critically broadens the scope of cued memory reactivations during sleep. On a neural basis slow oscillations, sharp wave-ripple complexes, sleep spindles and theta activity seem to be tightly linked to such successful reactivation processes. The results reviewed here provide a solid basis for future research and the development of technical applications, setting the stage for designing cueing of foreign vocabulary during sleep as an potential future technique in order to support vocabulary learning also in an educational setting. 


\section{Open Questions:}

What is the precise role of theta activity in the context of reactivation processes and how is their exact interplay with slow oscillations and sleep spindles?

How are the behavioural and oscillatory responses dependent on the pre-stimulus micro state, i.e. if cue presentation occurs during the up- or down state of the slow oscillations, inside or outside a sleep spindle, in phase or out-of phase of ongoing theta activity etc.?

What are the behavioural and oscillatory consequences of reactivation of foreign vocabulary during REM sleep? While odor-induced memory reactivation during REM sleep has revealed no memory benefits (Cordi et al., 2014; Rasch et al., 2007), some studies have reported hints for better memory performance after reactivation during REM sleep (Hars, Hennevin, \& Pasques, 1985; C. Smith \& Weeden, 1990; see Hennevin, Huetz, \& Edeline, 2007, for a review).

Does the degree of prior knowledge of closely related languages constitutes an important factor in determining the effectiveness of cueing during sleep? The close relationship of the languages used in our studies might have considerably affected the success of cueing during sleep in our studies. Hence the usage of more distant languages and the manipulation of the pre-sleep learning level in comparable studies might untangle the potential effects of prior knowledge, learning difficulty, and memory strength during encoding on cueing success.

Does cueing in a regular fashion affect sleep quality in an undesirable way? In our studies there was no indication that auditory cueing during sleep has any effect on sleep architecture. Thus on a macro level, sleep stages between experimental and control groups where highly 
comparable. Still, for future practical usage more fine-grained procedures would be needed in order to avoid the involuntary awakenings through auditory stimulation during sleep.

\section{Acknowledgements}

This work was supported by a grant from the Swiss National Foundation (SNF)

(100014_162388) and the research pool of the University of Fribourg. We want to thank

Maurice Göldi and Mick Lehmann for useful comments on earlier versions of the manuscript.

\section{References:}

Andrade, K. C., Spoormaker, V. I., Dresler, M., Wehrle, R., Holsboer, F., Sämann, P. G., \& Czisch, M. (2011). Sleep spindles and hippocampal functional connectivity in human NREM sleep. The Journal of Neuroscience, 31(28), 10331-10339.

Antony, J. W., Gobel, E. W., O'Hare, J. K., Reber, P. J., \& Paller, K. a. (2012). Cued memory reactivation during sleep influences skill learning. Nature Neuroscience, 15(8), 1114-6.

Arbon, E. L., Knurowska, M., \& Dijk, D.-J. (2015). Randomised clinical trial of the effects of prolonged-release melatonin, temazepam and zolpidem on slow-wave activity during sleep in healthy people. Journal of Psychopharmacology, 29(7), 764-76.

Axmacher, N., Mormann, F., Fernández, G., Elger, C. E., \& Fell, J. (2006). Memory formation by neuronal synchronization. Brain Research Reviews, 52(1), 170-82.

Bakker, I., Takashima, A., Hell, J. G. van, Janzen, G., \& McQueen, J. M. (2015). Changes in Theta and Beta Oscillations as Signatures of Novel Word Consolidation. Journal of Cognitive Neuroscience. 27(7):1286-97

Barnes, D. C., \& Wilson, D. A. (2014). Slow-wave sleep-imposed replay modulates both strength and precision of memory. The Journal of Neuroscience, 34(15), 5134-42.

Bastuji, H., \& García-Larrea, L. (1999). Evoked potentials as a tool for the investigation of human sleep. Sleep Medicine Reviews, 3(1), 23-45.

Battaglia, F. P., Sutherland, G. R., \& McNaughton, B. L. (2004). Hippocampal sharp wave bursts coincide with neocortical "up-state" transitions. Learning \& Memory, 11(6), 697704.

Batterink, L. J., Oudiette, D., Reber, P. J., \& Paller, K. A. (2014). Sleep facilitates learning a new linguistic rule. Neuropsychologia, 65C, 169-179. 4

Batterink, L. J., \& Paller, K. A. (2015). Sleep-based memory processing facilitates grammatical generalization: Evidence from targeted memory reactivation. Brain and Language. http://doi.org/10.1016/j.bandl.2015.09.003

Benchenane, K., Peyrache, A., Khamassi, M., Tierney, P. L., Gioanni, Y., Battaglia, F. P., \& Wiener, S. I. (2010). Coherent Theta Oscillations and Reorganization of Spike Timing in the Hippocampal- Prefrontal Network upon Learning. Neuron, 66(6), 921-936.

Bendor, D., \& Wilson, M. a. (2012). Biasing the content of hippocampal replay during sleep. Nature Neuroscience, 15(10), 1439-44.

Bergmann, T. O., Mölle, M., Diedrichs, J., Born, J., \& Siebner, H. R. (2012). Sleep spindle- 
related reactivation of category-specific cortical regions after learning face-scene associations. NeuroImage, 59(3), 2733-42.

Born, J., \& Wilhelm, I. (2012). System consolidation of memory during sleep. Psychological Research, 76(2), 192-203.

Brualla, J., Romero, M. F., Serrano, M., \& Valdizán, J. R. (1998). Auditory event-related potentials to semantic priming during sleep. Electroencephalography and Clinical Neurophysiology, 108(3), 283-90.

Brunner, D. P., Dijk, D. J., Münch, M., \& Borbély, a a. (1991). Effect of zolpidem on sleep and sleep EEG spectra in healthy young men. Psychopharmacology, 104(1), 1-5.

Cairney, S. A, Durrant, S. J., Hulleman, J., \& Lewis, P. A. (2014). Targeted memory reactivation during slow wave sleep facilitates emotional memory consolidation. Sleep, 37(4), 701-7.

Carracedo, L. M., Kjeldsen, H., Cunnington, L., Jenkins, A., Schofield, I., Cunningham, M. O., Whittington, M. a. (2013). A neocortical delta rhythm facilitates reciprocal interlaminar interactions via nested theta rhythms. The Journal of Neuroscience, 33(26), $10750-61$.

Carskadon, M. A., \& Herz, R. S. (2004). Minimal olfactory perception during sleep: why odor alarms will not work for humans. Sleep, 27(3), 402-405.

Chu, S., \& Downes, J. J. (2002). Proust nose best: Odors are better cues of autobiographical memory. Memory \& Cognition, 30(4), 511-518.

Clemens, Z., Molle, M., Eross, L., Barsi, P., Halasz, P., \& Born, J. (2007). Temporal coupling of parahippocampal ripples, sleep spindles and slow oscillations in humans. Brain, 130(11), 2868-2878.

Coenen, A. M. L., \& Vendrik, A. J. H. (1972). Determination of the transfer ratio of cat's geniculate neurons through quasi-intracellular recordings and the relation with the level of alertness. Experimental Brain Research, 14(3), 227-242.

Colrain, I. M. (2005). The K-complex: a 7-decade history. Sleep, 28, 255-273.

Contreras, D., Destexhe, A., \& Steriade, M. (1997). Intracellular and computational characterization of the intracortical inhibitory control of synchronized thalamic inputs in vivo. Journal of Neurophysiology, 78(1), 335-350.

Cordi, M. J., Diekelmann, S., Born, J., \& Rasch, B. (2014). No effect of odor-induced memory reactivation during REM sleep on declarative memory stability. Frontiers in Systems Neuroscience, 8, 157.

Cousins, J. N., El-Deredy, W., Parkes, L. M., Hennies, N., \& Lewis, P. A. (2014). Cued Memory Reactivation during Slow-Wave Sleep Promotes Explicit Knowledge of a Motor Sequence. Journal of Neuroscience, 34(48), 15870-15876.

Cox, R., Hofman, W. F., de Boer, M., \& Talamini, L. M. (2014). Local sleep spindle modulations in relation to specific memory cues. NeuroImage, 99, 103-10.

Creery, J. D., Oudiette, D., Antony, J. W., \& Paller, K. A. (2015). Targeted Memory Reactivation during Sleep Depends on Prior Learning. Sleep, 38(5), 755-63.

Czisch, M., Wehrle, R., Stiegler, A., Peters, H., Andrade, K., Holsboer, F., \& Sämann, P. G. (2009). Acoustic oddball during NREM sleep: a combined EEG/fMRI study. PloS One, 4(8), e6749.

Dang-vu, T. T., Bonjean, M., Schabus, M., Boly, M., \& Darsaud, A. (2011). Interplay between spontaneous and induced brain activity during human non-rapid eye movement sleep. Proceedings of the National Academy of Sciences of the United States of America, 108(37), 15438-43

Dave, A., \& Marholiash, D. (2000). Song Replay During Sleep and Computational Rules for 
Sensorimotor Vocal Learning. Science, 290(5492), 812-816.

Davis, M. H., \& Gaskell, M. G. (2009). A complementary systems account of word learning: neural and behavioural evidence. Philosophical Transactions of the Royal Society of London. Series B, Biological Sciences, 364(1536), 3773-800.

Destexhe, A., Hughes, S. W., Rudolph, M., \& Crunelli, V. (2007). Are corticothalamic "up"states fragments of wakefulness? Trends in Neurosciences. 30(7):334-42

Diekelmann, S. (2014). Sleep for cognitive enhancement. Frontiers in Systems Neuroscience, $8,46$.

Diekelmann, S., \& Born, J. (2010). The memory function of sleep. Nature Reviews. Neuroscience, 11(2), 114-26.

Diekelmann, S., \& Born, J. (2015). Cueing fear memory during sleep--to extinguish or to enhance fear? Sleep, 38(3), 337-9.

Diekelmann, S., Büchel, C., Born, J., \& Rasch, B. (2011). Labile or stable: opposing consequences for memory when reactivated during waking and sleep. Nature Neuroscience, 14(3), 381-386.

Dongen, E. V. Van, Takashima, A., Barth, M., Zapp, J., Schad, L. R., \& Paller, K. A. (2012). Memory stabilization with targeted reactivation during human slow-wave sleep. Proceedings of the National Academy of Sciences of the United States of America, 109(26):10575-80.

Dongen, E. V. Van, Thielen, J., Takashima, A., \& Barth, M. (2012). Sleep Supports Selective Retention of Associative Memories Based on Relevance for Future Utilization, PLoS One 7(8):e43426. http://doi.org/10.1371/journal.pone.0043426

Dumay, N., \& Gaskell, M. G. (2007). Sleep-associated changes in the mental representation of spoken words. Psychological Science, 18(1), 35-9.

Edeline, J. M., Dutrieux, G., Manunta, Y., \& Hennevin, E. (2001). Diversity of receptive field changes in auditory cortex during natural sleep. The European Journal of Neuroscience, $14,1865-1880$.

Ellenbogen, J. M., Hu, P. T., Payne, J. D., Titone, D., \& Walker, M. P. (2007). Human relational memory requires time and sleep. Proceedings of the National Academy of Sciences of the United States of America, 104, 7723-7728.

Feld, G. B., Wilhelm, I., Ma, Y., Groch, S., Binkofski, F., Mölle, M., \& Born, J. (2013). Slow wave sleep induced by GABA agonist tiagabine fails to benefit memory consolidation. Sleep, 36(9), 1317-26.

Fenn, K. M., Nusbaum, H. C., \& Margoliash, D. (2003). Consolidation during sleep of perceptual learning of spoken language. Nature, 425(6958):614-6.

Friedrich, M., Wilhelm, I., Born, J., \& Friederici, A. D. (2015). Generalization of word meanings during infant sleep. Nature Communications, 6, 6004.

Fuentemilla, L., Miró, J., Ripollés, P., Vilà-Balló, A., Juncadella, M., Castañer, S., Rodríguez-Fornells, A. (2013). Hippocampus-dependent strengthening of targeted memories via reactivation during sleep in humans. Current Biology, 23(18), 1769-75.

Fuentemilla, L., Penny, W. D., Cashdollar, N., Bunzeck, N., \& Düzel, E. (2010). ThetaCoupled Periodic Replay in Working Memory. Current Biology, 20, 606-612.

Gabrieli, J. D. (1998). Cognitive neuroscience of human memory. Annual Review of Psychology, 49, 87-115.

Gais, S., \& Born, J. (2004). Declarative memory consolidation: mechanisms acting during human sleep. Learning \& Memory, 11(6), 679-85.

Gais, S., Lucas, B., \& Born, J. (2006). Sleep after learning aids memory recall. Learning \& 
Memory, 13(3), 259-62.

Gaskell, M. G., Warker, J., Lindsay, S., Frost, R., Guest, J., Snowdon, R., \& Stackhouse, A. (2014). Sleep Underpins the Plasticity of Language Production. Psychological Science, $25,1457-1465$.

Genzel, L., Kroes, M. C. W., Dresler, M., \& Battaglia, F. P. (2014). Light sleep versus slow wave sleep in memory consolidation: a question of global versus local processes? Trends in Neurosciences, 37(1), 10-9.

Godden, D. R., \& Baddeley, A. D. (1975). Context-dependent memory in two natural environments: on land and underwater. British Journal of Psychology, 66(3), 325-331.

Gómez, R. L., Bootzin, R. R., \& Nadel, L. (2006). Naps promote abstraction in languagelearning infants. Psychological Science, 17(8), 670-4.

Hall-Porter, J. M., Schweitzer, P. K., Eisenstein, R. D., Ahmed, H. A. H., \& Walsh, J. K. (2014). The effect of two benzodiazepine receptor agonist hypnotics on sleep-dependent memory consolidation. Journal of Clinical Sleep Medicine, 10(1), 27-34.

Hars, B., Hennevin, E., \& Pasques, P. (1985). Improvement of learning by cueing during postlearning paradoxical sleep. Behavioural Brain Research, 18(3), 241-50.

Hauner, K. K., Howard, J. D., Zelano, C., \& Gottfried, J. A. (2013). Stimulus-specific enhancement of fear extinction during slow-wave sleep. Nature Neuroscience, 16(11),

Havekes, R., Meerlo, P., \& Abel, T. (2015). Animal studies on the role of sleep in memory: from behavioral performance to molecular mechanisms. Current Topics in Behavioral Neurosciences, 25, 183-206.

He, J., Sun, H.-Q., Li, S.-X., Zhang, W.-H., \& Shi, J. (2015). Effect of Conditioned Stimulus Exposure during Slow Wave Sleep on Fear Memory Extinction in Humans. Sleep, 38(3), 423-431.

Henderson, L. M., Weighall, A. R., Brown, H., \& Gareth Gaskell, M. (2012). Consolidation of vocabulary is associated with sleep in children. Developmental Science, 15(5), 67487.

Hennevin, E., Huetz, C., \& Edeline, J. M. (2007). Neural representations during sleep: From sensory processing to memory traces. Neurobiology of Learning and Memory, 87(3), 416-440.

Hot, P., Rauchs, G., Bertran, F., Denise, P., Desgranges, B., Clochon, P., \& Eustache, F. (2011). Changes in sleep theta rhythm are related to episodic memory impairment in early Alzheimer's disease. Biological Psychology, 87(3), 334-339.

Hu, X., Antony, J. W., Creery, J. D., Vargas, I. M., Bodenhausen, G. V., \& Paller, K. A. (2015). Unlearning implicit social biases during sleep. Science, 348(6238), 1013-1015.

Huerta, P. T., \& Lisman, J. E. (1995). Bidirectional synaptic plasticity induced by a single burst during cholinergic theta oscillation in CA1 in vitro. Neuron, 15, 1053-1063.

Hyman, J. M., Wyble, B. P., Goyal, V., Rossi, C. A., \& Hasselmo, M. E. (2003). Stimulation in hippocampal region CA1 in behaving rats yields long-term potentiation when delivered to the peak of theta and long-term depression when delivered to the trough. The Journal of Neuroscience, 23, 11725-11731.

Ibáñez, A., López, V., \& Cornejo, C. (2006). ERPs and contextual semantic discrimination: degrees of congruence in wakefulness and sleep. Brain and Language, 98(3), 264-275.

Isomura, Y., Sirota, A., Ozen, S., Montgomery, S., Mizuseki, K., Henze, D. A., \& Buzsáki, G. (2006). Integration and segregation of activity in entorhinal-hippocampal subregions by neocortical slow oscillations. Neuron, 52(5), 871-82.

Issa, E. B., \& Wang, X. (2008). Sensory responses during sleep in primate primary and secondary auditory cortex. The Journal of Neuroscience, 28, 14467-14480. 
Jacobs, J., Hwang, G., Curran, T., \& Kahana, M. J. (2006). EEG oscillations and recognition memory: theta correlates of memory retrieval and decision making. NeuroImage, 32(2), 978-87.

Jenkins, J. G., \& Dallenbach, K. M. (1924). Obliviscence during sleep and waking. The American Journal of Psychology, 35, 605-602.

Ji, D., \& Wilson, M. a. (2007). Coordinated memory replay in the visual cortex and hippocampus during sleep. Nature Neuroscience, 10(1), 100-7.

Kaestner, E. J., Wixted, J. T., \& Mednick, S. C. (2013). Pharmacologically increasing sleep spindles enhances recognition for negative and high-arousal memories. Journal of Cognitive Neuroscience, 25(10), 1597-610.

Karakaş, S., Cakmak, E. D., Bekçi, B., \& Aydin, H. (2007). Oscillatory responses representing differential auditory processing in sleep. International Journal of Psychophysiology, 65(1), 40-50.

Klimesch, W., Doppelmayr, M., Stadler, W., Pöllhuber, D., Sauseng, P., \& Röhm, D. (2001). Episodic retrieval is reflected by a process specific increase in human electroencephalographic theta activity. Neuroscience Letters, 302(1), 49-52.

Klimesch, W., Hanslmayr, S., Sauseng, P., Gruber, W., Brozinsky, C. J., Kroll, N. E. A., ... Doppelmayr, M. (2006). Oscillatory EEG correlates of episodic trace decay. Cerebral Cortex, 16, 280-290.

Larson, J., Wong, D., \& Lynch, G. (1986). Patterned stimulation at the theta frequency is optimal for the induction of hippocampal long-term potentiation. Brain Research, 368(2), 347-350.

Lewis, P. A., \& Durrant, S. J. (2011). Overlapping memory replay during sleep builds cognitive schemata. Trends in Cognitive Sciences. 15(8):343-51.

Lisman, J. E., \& Jensen, O. (2013). The Theta-Gamma Neural Code. Neuron, 77(6), 10021016.

Marr, D. (1971). Simple memory: a theory for archicortex. Philosophical Transactions of the Royal Society of London. Series B, Biological Sciences, 262, 23-81.

Marshall, L., Helgadóttir, H., Mölle, M., \& Born, J. (2006). Boosting slow oscillations during sleep potentiates memory. Nature. 444(7119):610-3.

McClelland, J. L., McNaughton, B. L., \& O'Reilly, R. C. (1995). Why there are complimentary learning systems in the hippocampus and neocortex: Insights from the successes and failures of connectionist models of learning and memory. Psychol. Rev., $102,419-457$.

McCormick, D. A., \& Bal, T. (1994). Sensory gating mechanisms of the thalamus. Current Opinion in Neurobiology. 4(4):550-6.

McGaugh, J. L. (2000). Memory--a century of consolidation. Science, 287(5451), 248-51.

Mednick, S. C., McDevitt, E. A., Walsh, J. K., Wamsley, E., Paulus, M., Kanady, J. C., \& Drummond, S. P. A. (2013). The critical role of sleep spindles in hippocampaldependent memory: a pharmacology study. The Journal of Neuroscienc, 33(10), 4494504.

Melendez, J., Galli, I., Boric, K., Ortega, A., Zuniga, L., Henriquez-Roldan, C. F., \& Cardenas, A. M. (2005). Zolpidem and triazolam do not affect the nocturnal sleepinduced memory improvement. Psychopharmacology, 181(1), 21-26.

Menz, M. M., Rihm, J. S., Salari, N., Born, J., Kalisch, R., Pape, H. C., Büchel, C. (2013). The role of sleep and sleep deprivation in consolidating fear memories. NeuroImage, 75 , 87-96.

Mölle, M., Bergmann, T. O., Marshall, L., \& Born, J. (2011). Fast and slow spindles during 
the sleep slow oscillation: disparate coalescence and engagement in memory processing. Sleep, 34(10), 1411-21.

Mölle, M., Eschenko, O., Gais, S., Sara, S. J., \& Born, J. (2009). The influence of learning on sleep slow oscillations and associated spindles and ripples in humans and rats. European Journal of Neuroscience, 29, 1071-1081.

Mölle, M., Marshall, L., Gais, S., \& Born, J. (2002). Grouping of spindle activity during slow oscillations in human non-rapid eye movement sleep. The Journal of Neuroscience, 22(24), 10941-7.

Mormann, F., Fell, J., Axmacher, N., Weber, B., Lehnertz, K., Elger, C. E., \& Fernández, G. (2005). Phase/amplitude reset and theta-gamma interaction in the human medial temporal lobe during a continuous word recognition memory task. Hippocampus, 15(7), 890-900.

Murakami, M., Kashiwadani, H., Kirino, Y., \& Mori, K. (2005). State-dependent sensory gating in olfactory cortex. Neuron, 46, 285-296.

Nader, K., \& Hardt, O. (2009). A single standard for memory: the case for reconsolidation. Nature Reviews. Neuroscience, 10(3), 224-34.

Ngo, H. V. V, Claussen, J. C., Born, J., \& Mölle, M. (2013). Induction of slow oscillations by rhythmic acoustic stimulation. Journal of Sleep Research, 22(1), 22-31.

Ngo, H.-V. V, Martinetz, T., Born, J., \& Mölle, M. (2013). Auditory Closed-Loop Stimulation of the Sleep Slow Oscillation Enhances Memory. Neuron, 78(3):545-53.

Ngo, H.-V. V., Miedema, A., Faude, I., Martinetz, T., Molle, M., \& Born, J. (2015). Driving Sleep Slow Oscillations by Auditory Closed-Loop Stimulation--A Self-Limiting Process. Journal of Neuroscience, 35(17).

Nieuwenhuis, I. L. C., Folia, V., Forkstam, C., Jensen, O., \& Petersson, K. M. (2013). Sleep Promotes the Extraction of Grammatical Rules. PLoS ONE, 8(6):e65046.

Nir, Y., Vyazovskiy, V. V, Cirelli, C., Banks, M. I., \& Tononi, G. (2015). Auditory Responses and Stimulus-Specific Adaptation in Rat Auditory Cortex are Preserved Across NREM and REM Sleep. Cerebral Cortex, 25(5):1362-78.

Nyhus, E., \& Curran, T. (2010). Functional role of gamma and theta oscillations in episodic memory. Neuroscience and Biobehavioral Reviews, 34(7), 1023-35.

O’Neill, J., Pleydell-Bouverie, B., Dupret, D., \& Csicsvari, J. (2010). Play it again: reactivation of waking experience and memory. Trends in Neurosciences, 33(5), 220 229.

Staresina, B. P., Ole Bergmann, T., Bonnefond, M., van der Meij, R., Jensen, O., Deuker, L., Elger, C. E., Axmacher, N,. Fell, J. (2015). Hierarchical nesting of slow oscillations, spindles and ripples in the human hippocampus during sleep. Nature Neuroscience, $18(11), 1679-86$.

Osipova, D., Takashima, A., Oostenveld, R., Fernández, G., Maris, E., \& Jensen, O. (2006). Theta and gamma oscillations predict encoding and retrieval of declarative memory. The Journal of Neuroscience 26(28), 7523-31.

Oudiette, D., \& Paller, K. A. (2013). Upgrading the sleeping brain with targeted memory reactivation. Trends in Cognitive Sciences, 17(3):142-9

Pavlides, C., \& Winson, J. (1989). Influences of hippocampal place cell firing in the awake state on the activity of these cells during subsequent sleep episodes. The Journal of Neuroscience, 9(8), 2907-18.

Peigneux, P., Laureys, S., Fuchs, S., Collette, F., Perrin, F., Reggers, J., Maquet, P. (2004). Are spatial memories strengthened in the human hippocampus during slow wave sleep? Neuron, 44(3), 535-45. 
Pena, J. L., Perez-Perera, L., Bouvier, M., \& Velluti, R. A. (1999). Sleep and wakefulness modulation of the neuronal firing in the auditory cortex of the guinea pig. Brain Research, 816, 463-470.

Pennartz, C. M. A., Lee, E., Verheul, J., Lipa, P., Barnes, C. A., \& McNaughton, B. L. (2004). The ventral striatum in off-line processing: ensemble reactivation during sleep and modulation by hippocampal ripples. The Journal of Neuroscience, 24, 6446-6456.

Perrin, F., Bastuji, H., \& Garcia-Larrea, L. (2002). Detection of verbal discordances during sleep. Neuroreport, 13(10), 1345-9.

Perrin, F., García-Larrea, L., Mauguière, F., \& Bastuji, H. (1999). A differential brain response to the subject's own name persists during sleep. Clinical Neurophysiology, $110,2153-2164$.

Peyrache, A., Battaglia, F. P., \& Destexhe, A. (2011). Inhibition recruitment in prefrontal cortex during sleep spindles and gating of hippocampal inputs, Proceedings of the National Academy of Sciences of the United States of America, 108(41):17207-12.

Peyrache, A., Khamassi, M., Benchenane, K., Wiener, S. I., \& Battaglia, F. P. (2009). Replay of rule-learning related neural patterns in the prefrontal cortex during sleep. Nature Neuroscience, 12, 919-926.

Pinker, S. (2000). Survival of the clearest. Nature, 404(6777):441-2.

Plihal, W., \& Born, J. (1995). Effects of Early and Late Nocturnal Sleep on Declarative and Procedural Memory, Journal of Cognitive Neuroscience, 9(4):534-47.

Plihal, W., \& Born, J. (1999). Effects of early and late nocturnal sleep on priming and spatial memory. Psychophysiology, 36, 571-582.

Portas, C. M., Krakow, K., Allen, P., Josephs, O., Armony, J. L., \& Frith, C. D. (2000). Auditory processing across the sleep-wake cycle: simultaneous EEG and fMRI monitoring in humans. Neuron, 28(3), 991-9.

Pratt, H., Berlad, I., \& Lavie, P. (1999). "Oddball” event-related potentials and information processing during REM and non-REM sleep. Clinical Neurophysiology, 110, 53-61.

Rasch, B., \& Born, J. (2007). Maintaining memories by reactivation. Current Opinion in Neurobiology, 17(6), 698-703.

Rasch, B., \& Born, J. (2013). About Sleep's Role in Memory. Physiological Reviews, 93(2), 681-766.

Rasch, B., Büchel, C., Gais, S., \& Born, J. (2007). Odor cues during slow-wave sleep prompt declarative memory consolidation. Science, 315(5817), 1426-1429.

Rattenborg, N. C., Martinez-Gonzalez, D., Roth, T. C., \& Pravosudov, V. V. (2011). Hippocampal memory consolidation during sleep: a comparison of mammals and birds. Biological Reviews of the Cambridge Philosophical Society, 86(3), 658-691.

Rihm, J. S., Diekelmann, S., Born, J., \& Rasch, B. (2014). Reactivating memories during sleep by odors: odor specificity and associated changes in sleep oscillations. Journal of Cognitive Neuroscience, 26, 1806-1818.

Rihm, J. S., \& Rasch, B. (2015). Replay of conditioned stimuli during late REM and stage N2 sleep influences affective tone rather than emotional memory strength. Neurobiology of Learning and Memory, 122, 142-151.

Ritter, S. M., Strick, M., Bos, M. W., Van Baaren, R. B., \& Dijksterhuis, A. (2012). Good morning creativity: task reactivation during sleep enhances beneficial effect of sleep on creative performance. Journal of Sleep Research, 21(6):643-7.

Rolls, A., Makam, M., Kroeger, D., Colas, D., Lecea, L. De, \& Heller, H. C. (2013). Sleep to forget : interference of fear memories during sleep. Molecular Psychiatry, 18(11), 11661170 . 
Rudoy, J. D., Voss, J. L., Westerberg, C. E., \& Paller, K. A. (2009). Strengthening individual memories by reactivating them during sleep. Science, 326(5956), 1079.

Schabus, M., Hoedlmoser, K., Pecherstorfer, T., \& Kloesch, G. (2005). Influence of Midday Naps on Declarative Memory Performance and Motivation. Somnologie, 9, 148-153.

Schönauer, M., Geisler, T., \& Gais, S. (2013). Strengthening Procedural Memories by Reactivation in Sleep. Journal of Cognitive Neuroscience, 26(1):143-5.

Schreiner, T., Göldi, M., \& Rasch, B. (2015). Cueing vocabulary during sleep increases theta activity during later recognition testing. Psychophysiology, 52(11), 1538-43.

Schreiner, T., Lehmann, M., \& Rasch, B. (2015). Auditory feedback blocks memory benefits of cueing during sleep. Nature Communications, 6, 8729.

Schreiner, T., \& Rasch, B. (2015a). Boosting Vocabulary Learning by Verbal Cueing During Sleep. Cerebral Cortex, 25(11), 4169-79.

Schreiner, T., \& Rasch, B. (2015b). Cueing vocabulary in awake subjects during the day has no effect on memory. Somnologie - Schlafforschung Und Schlafmedizin, 19(2), 133140.

Siapas, A. G., \& Wilson, M. A. (1998). Coordinated interactions between hippocampal ripples and cortical spindles during slow-wave sleep. Neuron, 21, 1123-1128.

Sirota, A., \& Buzsáki, G. (2005). Interaction between neocortical and hippocampal networks via slow oscillations. Thalamus and Related Systems, 3(04), 245.

Sirota, A., Csicsvari, J., Buhl, D., \& Buzsáki, G. (2003). Communication between neocortex and hippocampus during sleep in rodents. Proceedings of the National Academy of Sciences of the United States of America, 100, 2065-2069.

Skaggs, W. E., \& McNaughton, B. L. (1996). Replay of neuronal firing sequences in rat hippocampus during sleep following spatial experience. Science, 271, 1870-1873.

Smith, C., \& Weeden, K. (1990). Post training REMs coincident auditory stimulation enhances memory in humans. Psychiatric Journal of the University of Ottawa, 15(2), 85-90.

Smith, S. M., \& Vela, E. (2001). Environmental context-dependent memory: A review and meta-analysis. Psychonomic Bulletin \& Review, 8(2), 203-220.

Steriade, M. (2006). Grouping of brain rhythms in corticothalamic systems. Neuroscience, 137, 1087-1106.

Steriade, M., \& Timofeev, I. (2003). Neuronal plasticity in thalamocortical networks during sleep and waking oscillations. Neuron. 37(4):563-76.

Stickgold, R., \& Walker, M. P. (2013). Sleep-dependent memory triage: evolving generalization through selective processing. Nature Neuroscience, 16(2), 139-45.

Tamminen, J., Payne, J. D., Stickgold, R., Wamsley, E. J., \& Gaskell, M. G. (2010). Sleep spindle activity is associated with the integration of new memories and existing knowledge. The Journal of Neuroscience, 30, 14356-14360.

Timofeev, I., \& Chauvette, S. (2011). Thalamocortical oscillations: local control of EEG slow waves. Current Topics in Medicinal Chemistry, 11(19), 2457-71.

Tononi, G., \& Cirelli, C. (2014). Sleep and the price of plasticity: from synaptic and cellular homeostasis to memory consolidation and integration. Neuron, 81(1), 12-34.

Trimper, J. B., Stefanescu, R. A., \& Manns, J. R. (2014). Recognition memory and thetagamma interactions in the hippocampus. Hippocampus, 24(3), 341-353.

Tronson, N. C., \& Taylor, J. R. (2007). Molecular mechanisms of memory reconsolidation. Nature Reviews. Neuroscience, 8(4), 262-75. 
Wagner, U., Gais, S., Haider, H., Verleger, R., \& Born, J. (2004). Sleep inspires insight, Nature, 427(6972):352-5.

Walker, M. P., \& Stickgold, R. (2004). Sleep-dependent learning and memory consolidation. Neuron, 44(1), 121-33.

Westerberg, C. E., Mander, B. A., Florczak, S. M., Weintraub, S., Mesulam, M.-M., Zee, P. C., \& Paller, K. A. (2012). Concurrent Impairments in Sleep and Memory in Amnestic Mild Cognitive Impairment. Journal of the International Neuropsychological Society, 18(3), 1-11.

Wilson, D. A., \& Sullivan, R. M. (2011). Cortical processing of odor objects. Neuron, 72(4):506-19.

Wilson, M. A., \& McNaughton, B. L. (1994). Reactivation of hippocampal ensemble memories during sleep. Science, 265, 676-679.

Ylinen, A, Bragin, A, Nádasdy, Z., Jandó, G., Szabó, I., Sik, A, \& Buzsáki, G. (1995). Sharp wave-associated high-frequency oscillation $(200 \mathrm{~Hz})$ in the intact hippocampus: network and intracellular mechanisms. The Journal of Neuroscience, 15, 30-46.

Figure1: Cueing foreign vocabulary during NREM sleep benefits memory consolidation.

A: Procedures: After studying 120 Dutch-German word pairs in the evening, participants slept for 3 hours or stayed awake during this time, depending on the experimental group. During the retention interval, parts of the Dutch words were repeatedly presented again for 90 minutes. After the retention interval, participants were tested on the German translation of the Dutch words using a cued recall procedure. $B$ : In the cueing sleep group, memory for the German translation of the cued Dutch words (black bar) was significantly improved when compared with uncued words (white bar). Recall of uncued words in the main sleep group was comparable with recall performance of words in the control sleep group, which did not receive any cues during sleep. $C+D$ : In contrast to cueing during NREM sleep, no enhancing effects of cueing on later memory retrieval occurred during wakefulness in both the active and the passive waking groups, neither during night-time nor during daytime wakefulness. $E+F$ : When presentation of Dutch words during sleep was immediately followed by correct and false feedback (i.e., correct or false German translation, grey bar), the beneficial effects on memory of cueing during sleep were completely blocked, as there was no difference in memory performance to uncued words (white bar). Again replay of single Dutch cues (black bar) significantly enhanced memory for the German translations. $G$ : For the control group a very similar enhancing effect appeared when Dutch cues were followed by delayed correct feedback (1500 ms instead of $200 \mathrm{~ms}$ ) as compared to uncued words (white bar) and Dutch cues which were immediately followed by a tone (grey bar). Retrieval performance is indicated as percentage of recalled German translations with performance before sleep set to $100 \%$. Values are mean \pm s.e.m. $* P \leq 0.05 ;+P<0.07$. 
A

Learning

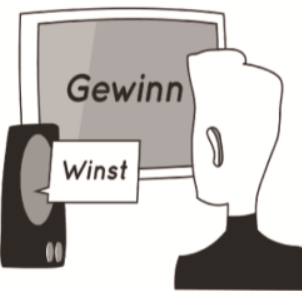

B

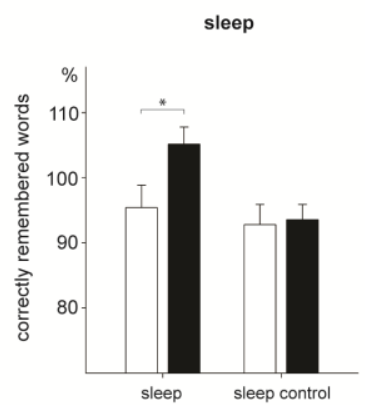

$\mathbf{E}$

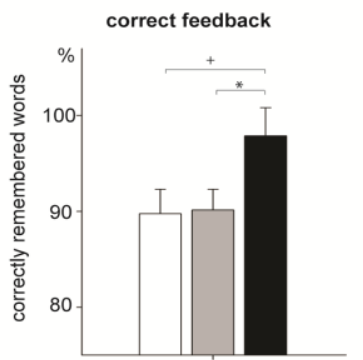

Reactivation

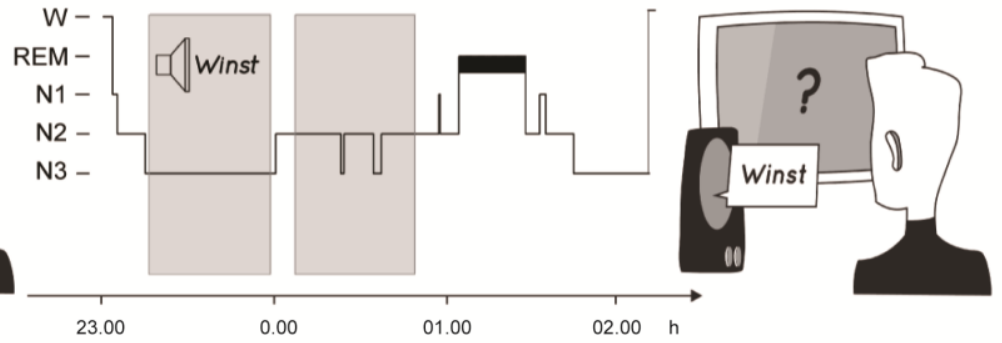

C

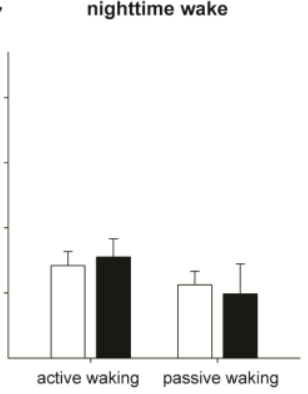

F

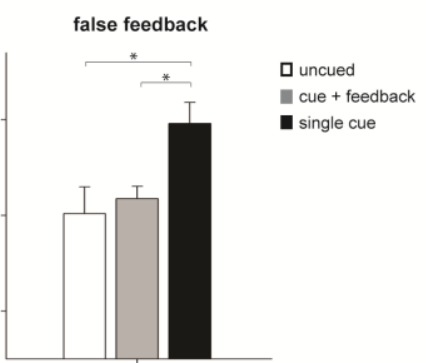

D daytime wake
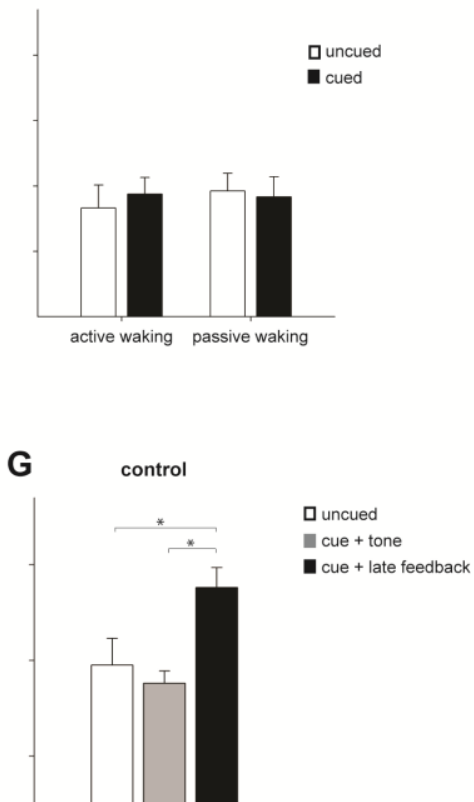

Figure 2: Effects of cueing on theta activity during later recognition testing during wakefulness.

A: Oscillatory activity recorded during recognition was computed for hits (words correctly identified as old) and correct rejections (words correctly identified as new). Theta power (representative electrode Pz) was significantly stronger for hits as compared to correct rejections. Bar charts indicate the number of significant electrodes over time. $B$ : To specifically determine potential effects of cueing, the total number of recognized old words was divided into old words replayed during sleep (cued) and old words not replayed during sleep (uncued). Cued old words elicited significantly stronger theta activity over centroparietal regions. $C$ : Theta power significantly differed between old and new words in source space (time window: $600-1,300 \mathrm{~ms}$ ). Activation was found in the left inferior prefrontal cortex, specifically Brodmann areas (BA) 47 and 45 (Broca's area). Activation is displayed 
on an anatomical template image on an axial, coronal and sagittal slice at a threshold of $p=$ 0.005 and cluster size of $\mathrm{k}>50$.

A

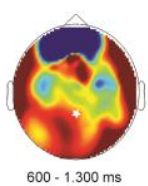

B

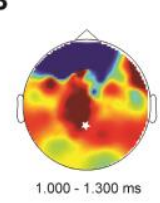

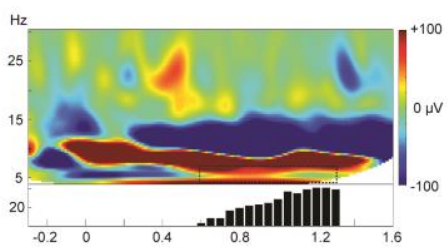

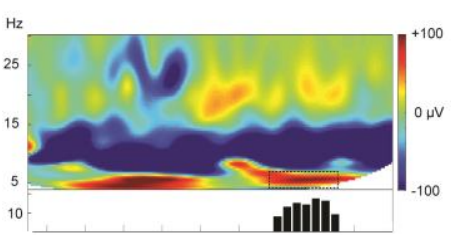

C

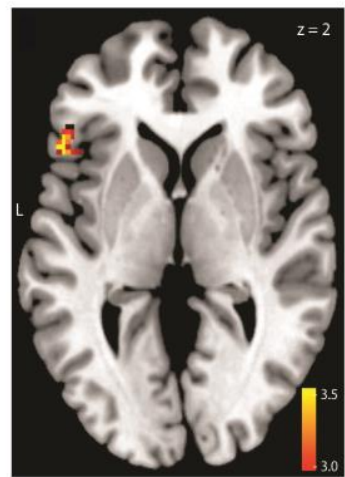

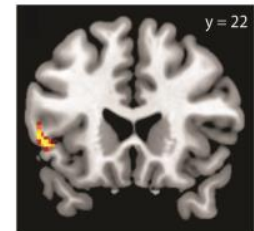

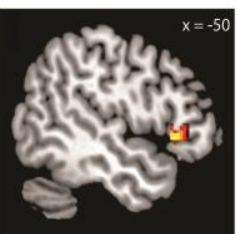

Figure 3: Working model and empirical findings for neural oscillatory mechanisms underlying the memory benefits of cueing during sleep. A: The presentation of a memoryrelated cue during NREM causes the hippocampus dependent reinstatement of the entire memory representations in the cortex, which is coded in theta and gamma activity. The thetagamma interaction provides the means for the temporal ordering and binding of individual memory representations. Sleep spindle occurring in parallel or shortly after increases in theta activity are necessary to support the stabilization, strengthening and integration of the reactivated memory representation during sleep.

$B$ : Disturbing these combined oscillatory signals by presenting further auditory stimuli in close proximity disrupts the reinstatement (theta, gamma) and stabilization / integration (spindle) process and blocks the beneficial effect of cueing during sleep. Delaying further auditory stimulation by a least 1.5 seconds after the cue is sufficient for a successful completion of the stabilization and integration process (not shown, see Figure 1G). $C$ : In our recent study (Schreiner, Lehmann, \& Rasch, 2015) successful cueing of Dutch words during NREM sleep was associated with enhanced power in the theta and spindle band (representative electrode F3). Verbal cues were presented at time $0 \mathrm{~ms}$. $D$ : The differences in the theta and spindle band vanished when Dutch cues were immediately followed by feedback. Importantly this effect was paralleled by a blockade of memory benefits of cueing on the behavioural level, indicating the critical role of theta and spindle activity for successful 
memory reactivations.
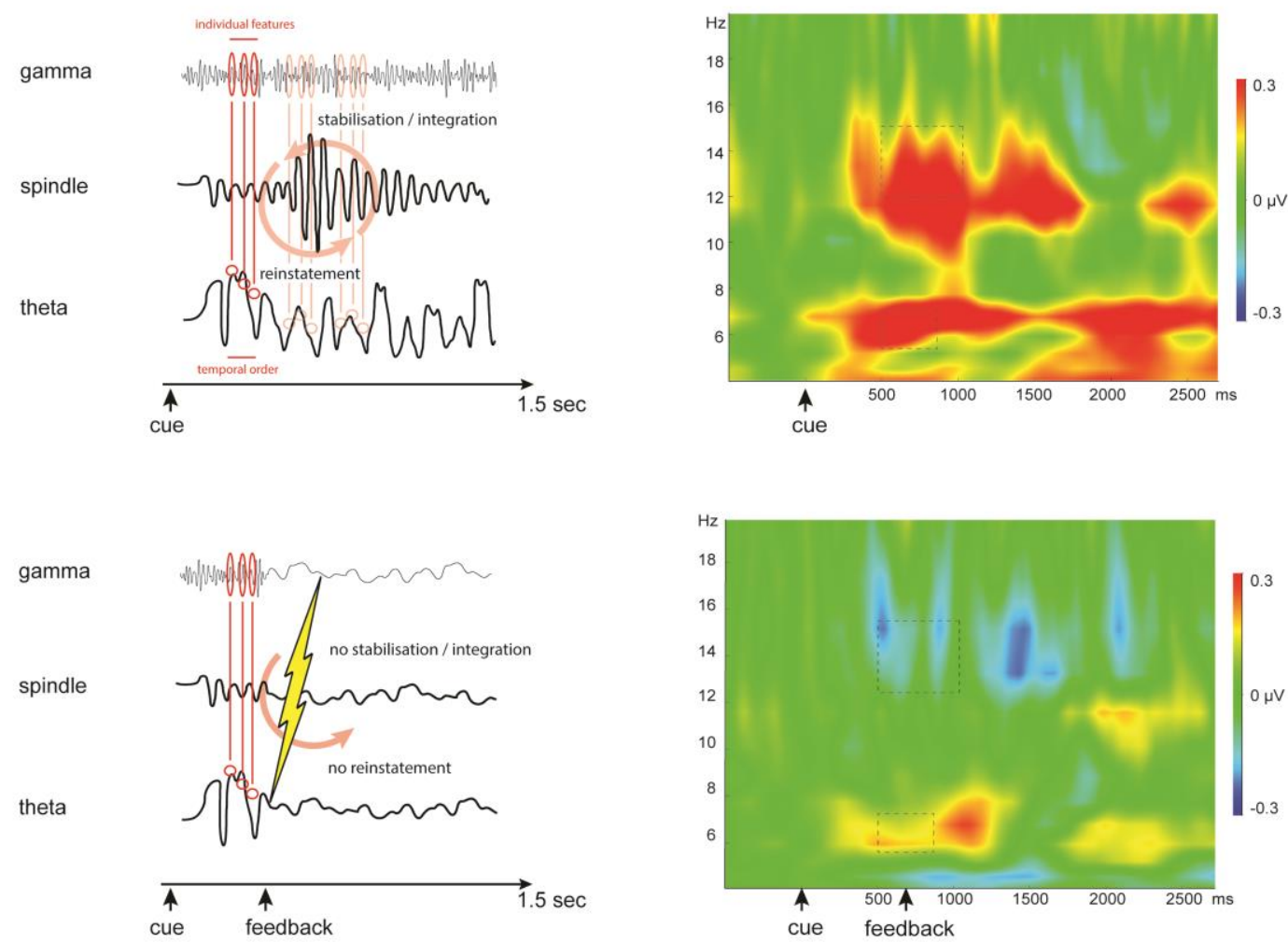